\title{
Submitted: Tltrasonographic inferior vena cava collapsibility Accepted: 08.07.2020 \\ Published: and distensibility indices for detecting the volume
status of critically ill pediatric patients
} 28.09.2020

\author{
Dincer Yildizdas, Nagehan Aslan
}

Department of Pediatric Intensive Care, Cukurova University Faculty of Medicine, Adana, Turkey

Correspondence: Nagehan Aslan, MD, Cukurova University, Faculty of Medicine, Adana, Turkey; tel.: +90505 54999 86, e-mail: nagehan_aslan@hotmail.com

DOI: $10.15557 / \mathrm{JoU} .2020 .0034$

\section{Keywords \\ inferior vena cava, pediatric, point-of-care ultrasound, volume status}

\begin{abstract}
Especially in recent years, the use of point-of-care ultrasound by non-radiologist clinicians has become widespread. Point-of-care ultrasound provides rapid responses to the problems of critically ill patients at the bedside. This technique has many important advantages, including being non-invasive, cheap, repeatable, painless, and radiation-free. Numerous studies have revealed the most important clinical benefits of point-of-care ultrasound use by pediatric intensive care providers. The inferior vena cava is a vessel that is highly sensitive to fluid changes. The inferior vena cava diameter can be measured by a point-of-care ultrasound, and represents a critical parameter in assessing the patient's fluid status. The inferior vena cava collapsibility index (in spontaneously breathing patients) and the inferior vena cava distensibility index (in mechanically ventilated patients) are calculated by determined formulas by using maximum and minimum diameters of the inferior vena cava. The indices are important guides for pediatric intensive care providers for managing their patients' fluid treatment. Although some authors claim it is not a reliable method, the technique is coming to fore in intensive care units day by day, and has an increasing trend among pediatric intensive care specialists. Here, we aim to give detailed information on the ultrasonographic inferior vena cava diameter measurement methods, and calculations of the inferior vena cava collapsibility index and inferior vena cava distensibility index, and emphasize the importance of a noninvasive, bedside, and objective method of detecting the volume status of critically ill patients for pediatric intensive care specialists according to the published literature.
\end{abstract}

\section{Introduction}

A point-of-care ultrasound (POCUS) is a type of bedside ultrasonographic assessment that is applied by the clinician in charge $\mathrm{e}^{(1)}$. It provides rapid and real-time answers about patients' clinical problems. The use of POCUS by clinicians has become common in recent years, especially in emergency and intensive care departments ${ }^{(2)}$. Around the world, the number of POCUS training courses intended for pediatric intensive care and emergency care specialists is increasing, and the newest generation of pediatricians has an interest in POCUS application. In fact, in recent years, POCUS has become part of the process of physically examining critically ill children in pediatric intensive care units (PICUs) ${ }^{(3)}$. Through POCUS results, clinicians are able to manage treatment options without requiring external consultation. The technique has many important advantages, for example it is easy to use, and can be repeated. It is also noninvasive, inexpensive, painless, and radiationfree ${ }^{(1)}$. Most pediatric intensive care societies, including the European Society of Pediatric and Neonatal Intensive Care (ESPNIC), provide advanced training courses, and encourage a new generation of pediatric intensive care specialists to use POCUS. The ESPNIC has also published international evidence-based guidelines on POCUS for neonates and children who are seriously ill. The organization has 


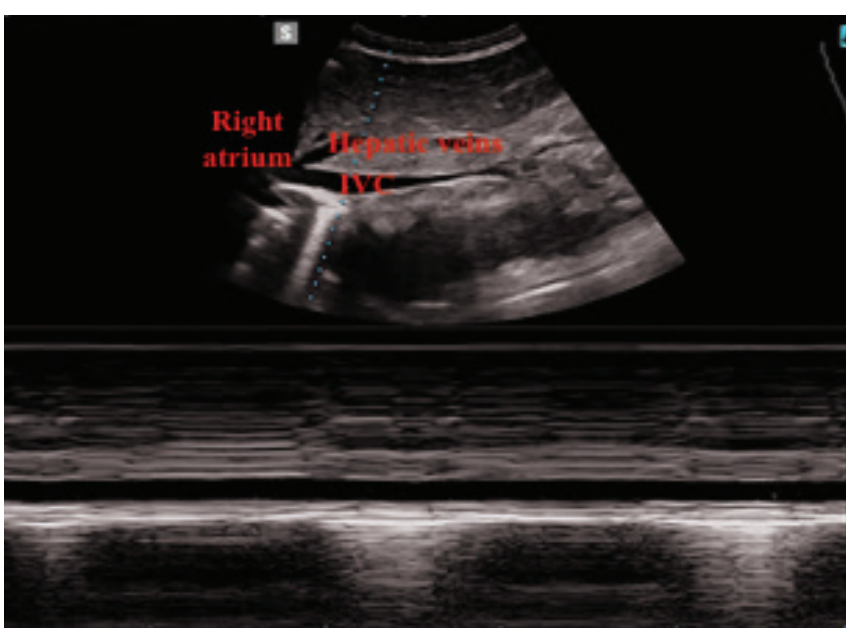

Fig. 1. M-Mode images of the inferior vena cava draining into the right atrium

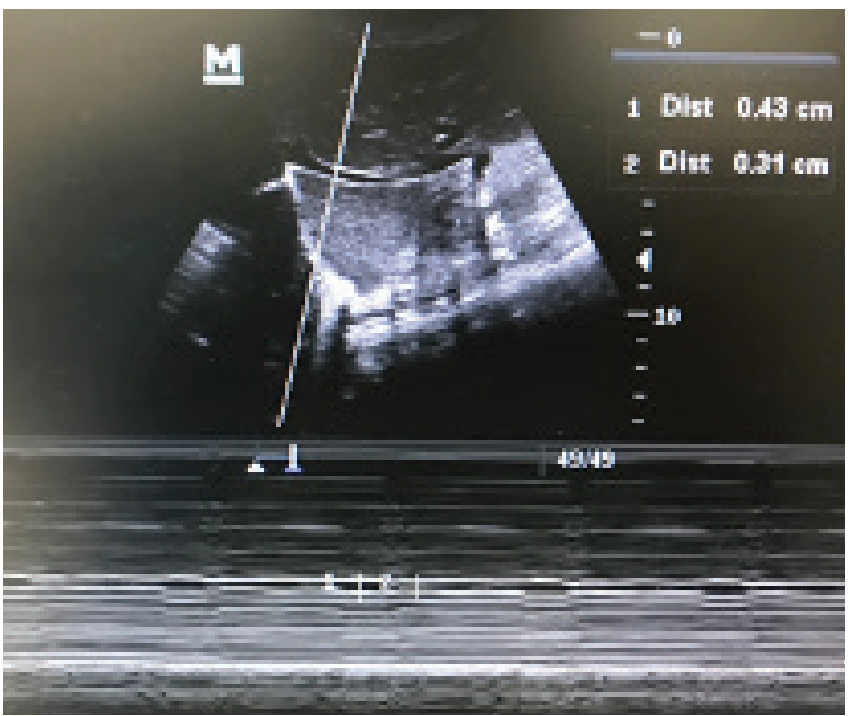

Fig. 2. Maximum and minimum diameter measurements of the inferior vena cava on breathing circulation IVC

recommended using POCUS in intensive care units based on strong evidence ${ }^{(4)}$.

There are different types of POCUS applications that are commonly used. These include critical-care echocardiography (to evaluate myocardial contractility and cardiac index measurements or detect pericardial tamponade), lung ultrasounds (to evaluate pneumothorax, pleural effusion and pneumonia, and ultrasound-guided thoracentesis), vascular ultrasounds (to insert a central venous catheter, peripheral venous catheter, or invasive arterial catheter), optic nerve sheath diameter measurements (for the clinical follow-up of increased intracranial pressure), fast intraabdominal assessments (to detect perihepatic or perisplenic hemorrhage), inferior vena cava (IVC) maximum and minimum diameter measurements (to evaluate the volume status of patients), confirmation of endotracheal tube and nasogastric tube placement, and management of cardiopulmonary resuscitation ${ }^{(5-12)}$.
Critically ill patients in PICUs frequently have critical and urgent problems. This patient group requires closer follow-up and needs quick assessments due to their hemodynamically unstable situation ${ }^{(13)}$. Detecting volume status and planning appropriate fluid therapy as well as inotropic, vasopressor, and inodilator treatments is vitally important, especially for patients in shock ${ }^{(13)}$. Appropriate fluid therapy is an important step in reducing the morbidity associated with multiple organ failure and mortality ${ }^{(14)}$. Skin turgor, heart rate, mean arterial pressure, urine output, and central venous pressure (CVP) are variables used to assess the patient's intravenous fluid status ${ }^{(15)}$. There are increasingly more studies, however, which suggest that the results obtained from these variables differ depending on personal assessments. This has raised the need for new methods to find more objective results about the volume status of critically ill children ${ }^{(16)}$.

The IVC is a vessel that is highly sensitive to fluid changes. It is collapsible, and varies in size depending on respiratory changes under intrathoracic pressure. During spontaneous breathing, the vessel closes on inspiration and opens on expiration. The IVC diameter can be measured by a POCUS quickly, non-invasively, and easily, and it is a critical parameter in assessing the patient's fluid status ${ }^{(17)}$. Several studies on adults have demonstrated that changes in the IVC diameter can be used to determine the patient's fluid status, but data obtained from children are still limited ${ }^{(18,19)}$.

In this article, we aim to give detailed information about IVC diameter measurement methods as well as calculations of the IVC collapsibility index (in spontaneously breathing patients) and the IVC distensibility index (in mechanically ventilated patients). We want to review the role of these measurements in the PICU setting, and emphasize the importance of a noninvasive, bedside, and objective method of detecting the volume status of critically ill patients for pediatric intensive care specialists based on the published literature.

\section{Ultrasonography technique for IVC measurements}

IVC collapsibility (in spontaneously breathing patients) and IVC distensibility (in mechanically ventilated patients) indices are measured by bedside ultrasonography. Measurements are performed while the patient is in the supine position. Generally, a $2-5-\mathrm{MHz}$ convex probe is used for the assessment. A sterile ultrasound gel is placed over the transducer at room temperature to obtain highquality images. IVC images are acquired in the sagittal section. To obtain a sagittal image, the probe is placed in the subxiphoid area and the liver is used as an acoustic window. Images of the IVC draining into the right atrium are obtained while the probe is in the subxiphoid area (Fig. 1). The minimum IVC diameter on inspiration and the maximum IVC diameter on expiration are recorded using M-mode just beyond the point where the hepatic veins drain into the IVC (Fig. 2). The maximum IVC diameter on inspiration and the minimum IVC diameter on expiration are measured using the same ultrasonographic method in intubated children for the vena cava distensibility index ${ }^{(19)}$. 
The IVC collapsibility index is calculated by the following formula: IVC collapsibility index $=[$ maximum diameter on expiration - (minimum diameter on inspiration/ maximum diameter on expiration) $]^{(20)}$. In mechanically ventilated patients, the IVC distensibility index is calculated using the formula: IVC distensibility index $=[$ (maximum diameter on inspiration -minimum diameter on expiration)/ minimum diameter on expiration] $]^{(21)}$.

\section{Pediatric literature on IVC collapsibility index}

Although there is a wide range of literature on adult patients, studies on pediatric patients are still limited, though an increasing number of studies look promising ${ }^{(19)}$. Again, pediatric data about these reference values are limited $^{(22)}$. Because of the lack of literature for pediatric age groups, the reference values defined for the IVC collapsibility index and the maximum and minimum diameters of IVC are derived from the adult population. In adults, an IVC collapsibility index of greater than $50 \%$ is associated with reduced right atrial pressure and severe dehydration, and indicates that the patient needs fluid therapy ${ }^{(23)}$. Mannarino et al. ${ }^{(24)}$ evaluated 516 healthy Italian children and gave reliable reference values for IVC diameters. In that study, the authors noticed that the reference value of the IVC collapsibility index was $30 \%$ for children older than one year, and $36 \%$ for children younger than one year. Another pediatric study aimed to obtain reference values for the IVCs in healthy children by evaluating a total of 475 Indian children from ages one to 12 . The authors gave reference values of the IVCs for the age groups and noticed a positive correlation between the maximum and minimum IVC diameters as well as somatic parameters like age, weight, height, and body surface $\operatorname{area}^{(25)}$. Kathuria et al. ${ }^{(26)}$ evaluated 63 children, aged zero to 22 months, without dehydration, in a pediatric emergency department. Their hypothesis was based on finding normative data for ultrasonographic IVC measurements. The authors detected a correlation between age and the minimum and maximum diameters of the IVC. A study by Kutty et al. (27) evaluated 120 healthy American children with a mean age of 8.3 years. They demonstrated a mean maximum IVC diameter of $12.1 \pm 3.8 \mathrm{~mm}$ and a mean minimum IVC diameter of $8.9 \pm 3.8 \mathrm{~mm}$ during spontaneous breathing. The IVC collapsibility index was 30 percent.

CVP is a standard of care for evaluating the fluid status in the PICU, and it is still commonly used in critical pediatric patients ${ }^{(15)}$. It reflects the right ventricular filling pressure, and provides information about the intravascular vol$u^{u m e}{ }^{(28)}$. However, the feasibility and efficacy of measuring CVP are often questioned because of risks associated with its invasive nature, such as infection, pneumothorax, and hemothorax, as well as commercial differences. These all depend on personal assessments ${ }^{(29)}$.

In recent years, more studies have found and emphasized the subjectivity and decreased reliability of CVP for detecting volume status ${ }^{(29)}$. The correlation between the IVC diameter and the right atrial pressure was first described in 1979 by Natori et al. ${ }^{(30)}$. Several adult studies have demonstrated that the IVC collapsibility index correlates well with $\operatorname{CVP}^{(18)}$. A study by Babaie et al. ${ }^{(20)}$, aimed at predicting the fluid status in pediatric patients, evaluated 70 children ranging in age from one month to 12 years. The authors reported a negative correlation between CVP and the IVC collapsibility index, and found the mean IVC collapsibility index to be $35 \pm 16 \%$. Another study by Mugloo et al. ${ }^{\left({ }^{31}\right)}$ evaluated 50 newborns and reported that the IVC collapsibility index and CVP were negatively correlated. In a study of 50 spontaneously breathing children aged five to 18 years, Vaish et al. ${ }^{(19)}$ reported a positive correlation of CVP with the IVC diameters both during inspiration and expiration, and a negative correlation with the collapsibility index. They found that the IVC collapsibility index better reflected the intravascular volume status at the beginning of fluid resuscitation, whereas the IVC diameter did this better at the end. Long et al. ${ }^{\left({ }^{(2)}\right)}$ performed a prospective observational study, with fluid bolus therapy applied, on 33 sepsis patients in a pediatric emergency department. They performed echocardiography after five and 60 minutes, followed by fluid bolus therapy, and detected that the IVC collapsibility index had poor test characteristics for predicting fluid responsiveness in spontaneously ventilating children with sepsis. Finally, Orso et al. ${ }^{\left({ }^{(3)}\right)}$ published a review article that comprised a total of 31 studies on IVC collapsibility, IVC distensibility, and IVC diameters. The review included three pediatric studies where the results revealed that an ultrasound evaluation of the IVC diameters and its respiratory variations did not seem to be a reliable method for predicting fluid responsiveness.

\section{Pediatric literature on IVC distensibility index}

Positive-pressure ventilation elevates the pleural and right atrial pressure values. It also reduces the venous return to the heart by increasing the intrathoracic pressure during inspiration. These factors act on the diameter and distensibility of the IVC. Finally, the IVC diameter dilates during inspiration and contracts during expiration in an intubated patient, unlike in spontaneously breathing patients ${ }^{(34)}$. Therefore, it is recommended that the IVC distensibility index be used instead of the IVC collapsibility index in patients undergoing positive-pressure mechanical ventilation ${ }^{(35)}$. Pediatric data are limited, and the IVC distensibility index is a new term in pediatric practice. For this reason, both Babaie et al. ${ }^{(20)}$ and Mugloo et al. ${ }^{(31)}$ used the IVC collapsibility index measurements in their studies on intubated patients under positive pressure. A study on mechanically ventilated adult patients in septic shock demonstrated that the IVC distensibility index values of greater than $18 \%$ were in favor of fluid deficit $^{(36)}$. Previous studies were conducted regarding the effectiveness of using the IVC distensibility index to predict fluid responsiveness in critically ill children ${ }^{(35)}$. In a pediatric study, Basu et al. ${ }^{(37)}$ compared the IVC distensibility index, CVP, and other common methods to assess the fluid status in mechanically ventilated pediatric patients that were seriously ill. There was no significant correlation between the IVC distensibility index and CVP. The study results showed that IVC distensibility had a good correlation with fluid overload, and might be useful to assess the degree of volume 
Tab. 1. The aim, formulas, benefits and question marks for the IVC collapsibility index and the IVC distensibility index

\begin{tabular}{|c|c|c|}
\hline & IVC collabsibility index & IVC distensibility index \\
\hline Aim & $\begin{array}{l}\text { Volume status assessment in spontaneously } \\
\text { breathing patients }\end{array}$ & $\begin{array}{l}\text { Volume status assessment in mechanically } \\
\text { ventilated patients }\end{array}$ \\
\hline \multirow[t]{2}{*}{ Method } & \multicolumn{2}{|c|}{$\begin{array}{l}\text { IVC images are acquired in the sagittal section. } \\
\text { Images of the IVC draining into the right atrium are obtained while the probe is in the subxiphoid area and the liver } \\
\text { is taken in the acoustic window. }\end{array}$} \\
\hline & $\begin{array}{l}\text { Minimum IVC diameter on inspiration and maximum IVC } \\
\text { diameter on expiration are measured. }\end{array}$ & $\begin{array}{l}\text { Maximum IVC diameter on inspiration and minimum IVC } \\
\text { diameter on expiration are measured. }\end{array}$ \\
\hline \multirow[t]{2}{*}{ Formula } & $\begin{array}{l}\text { (Maximum diameter on expiration - minimum diameter on } \\
\text { inspiration) }\end{array}$ & $\begin{array}{l}\text { Maximum diameter on inspiration - minimum diameter } \\
\text { on expiration }\end{array}$ \\
\hline & Maximum diameter on expiration & Minimum diameter on expiration \\
\hline Benefits & \multicolumn{2}{|c|}{ Quick, non-invasive, easy, repeatable, trustworthy and objective method for volume status evaluation ${ }^{17}$} \\
\hline \multirow{3}{*}{ Question marks } & \multicolumn{2}{|c|}{$\begin{array}{l}\text { There are no well-determined limits for the IVC collapsibility index and distensibility index in the pediatric age group } \\
\text { in the published literature. }\end{array}$} \\
\hline & \multicolumn{2}{|c|}{$\begin{array}{l}\text { Pediatric intensivists still use limitations described for adult patients for managing fluid therapy (for IVC collapsibility } \\
\text { index }>50 \% \text {, for IVC distensibility index }>18 \% \text { ). }\end{array}$} \\
\hline & \multicolumn{2}{|c|}{$\begin{array}{l}\text { Further studies on large groups of critically ill pediatric patients could help to determine ultrasonographically measured } \\
\text { pediatric IVC index limits }\end{array}$} \\
\hline
\end{tabular}

overload in patients. Bilgili et al. ${ }^{(35)}$ performed a prospective study with 24 pediatric urological surgery patients under general anesthesia. They measured the cardiac index, the IVC distensibility index, and the internal jugular vein distensibility index before and after a passive leg raising maneuver. Patients were termed fluid responders if $>10 \%$ increase was detected in the cardiac index after the maneuver. In the fluid responder group, the patients had higher IVC and internal jugular vein distensibility indices. The authors also noticed that the IVC distensibility index predicted fluid responsiveness in anesthetized pediatric patients. In an experimental animal study, Mendes et al. ${ }^{(38)}$ determined the accuracy of the IVC distensibility index for evaluating fluid responsiveness in rats with acute respiratory distress syndrome. They observed that an IVC distensibility index threshold of less than $25 \%$ was associated with a positive response to volume expansion. Although the results have been contradictory, and the reference values have not been described well yet, recent studies show that the IVC distensibility index is a reliable measure of predicting fluid responsiveness in mechanically ventilated children $^{(35)}$. A summary which includes the aim, formulas, benefits and question marks for the IVC collapsibility index and the IVC distensibility index is shown in Table 1.

\section{References}

1. O'Brien AJ, Brady RM: Point-of-care ultrasound in paediatric emergency medicine. J Paediatr Child Health 2016; 52: 174-180.

2. Moore CL, Copel JA: Point-of-care ultrasonography. N Engl J Med 2011; 364: 749-757.

3. Dubourg J, Javouhey E, Geeraerts T, Messerer M, Kassai B: Ultrasonography of optic nerve sheath diameter for detection of raised intracranial pressure: A systematic review and meta-analysis. Intensive Care Med 2011; 37: 1059-1068.

4. Singh Y, Tissot C, Fraga MV, Yousef N, Cortes RG, Lopez J et al.: International evidence-based guidelines on Point-of-Care Ultrasound (POCUS) for critically ill neonates and children issued by the POCUS

\section{Conclusion}

In conclusion, the collapsibility and distensibility indices of the IVC are ultrasonographic measurement methods that are noninvasive, quick, radiation-free, and can be done at the patient's bedside. Despite the lack of certain and reliable reference values of the IVC diameters as well as the IVC collapsibility and distensibility indices, an increasing number of studies with large patient groups will contribute to the literature on pediatric patients. Although there are opposing views, considering the lost prestige and reduced popularity of CVP, we believe that these measurement methods to evaluate the volume status of critical pediatric patients are increasingly coming to the fore in intensive care units.

\section{Conflict of interest}

Authors do not report any financial or personal connections with other persons or organizations, which might negatively affect the contents of this publication and/or claim authorship rights to this publication.
Working Group of the European Society of Paediatric and Neonatal Intensive Care (ESPNIC). Crit Care 2020; 24: 65.

5. Longjohn M, Wan J, Joshi V,Pershad J: Point-of-care echocardiography by pediatric emergency physicians. Pediatr Emerg Care 2011; 27: 693-696.

6. Xirouchaki N, Magkanas E, Vaporidi K, Kondili E, Plataki M, Patrianakos A et al.: Lung ultrasound in critically ill patients: comparison with bedside chest radiography. Intensive Care Med 2011; 37: 1488-1493.

7. Lamperti M, Bodenham AR, Pittiruti M, Blaivas M, Augoustides JG, Elbarbary $\mathrm{M}$ et al.: International evidence-based recommendations 
on ultrasound-guided vascular access. Intensive Care Med 2012; 38: $1105-1117$.

8. Dickman E, Tessaro MO, Arroyo AC, Haines LE, Marshall JP: Clinicianperformed abdominal sonography. Eur J Trauma Emerg Surg 2015; 41: 481-492.

9. Jauregui J, Nelson D, Choo E, Stearns B, Levine AC, Liebmann O et al.: The BUDDY (Bedside Ultrasound to Detect Dehydration in Youth) study. Crit Ultrasound J 2014; 6: 15.

10. Atalay YO, Polat AV, Ozkan EO, Tomak L, Aygun C, Tobias JD: Bedside ultrasonography for the confirmation of gastric tube placement in the neonate. Saudi J Anaesth 2019; 13: 23-27.

11. Blanco P, Buendía CM: Point-of-care ultrasound in cardiopulmonary resuscitation: a concise review. J Ultrasound 2017; 20: 193-198.

12. Singh P, Thakur A, Garg P, Aggarwal N, Kler N: Normative Data of Optimally Placed Endotracheal Tube by Point-of-care Ultrasound in Neonates. Indian Pediatr 2019; 56: 374-380.

13. Lee EP, Hsia SH, Lin JJ, Chan OW, Lee J, Lin CY et al.: Hemodynamic analysis of pediatric septic shock and cardiogenic shock using transpulmonary thermodilution. Biomed Res Int 2017; 2017: 3613475.

14. Goldstein B, Giroir B, Randolph A, International Consensus Conference on Pediatric Sepsis: International pediatric sepsis consensus conference: definitions for sepsis and organ dysfunction in pediatrics. Pediatr Crit Care Med 2005; 6: 2-8.

15. Dellinger RP, Levy MM, Rhodes A, Annane D, Gerlach H, Opal SM et al.: Surviving sepsis campaign: international guidelines for Management of Severe Sepsis and Septic Shock. Crit Care Med 2013; 41: $580-637$.

16. Leache Irigoyen J, Marín Corral J, Oliva Zelaya I, Moreno Muñoz G, Blàquez Alcaide V, Bodi Asaera A et al.: Accuracy of cardiac output estimations by transthoracic echocardiography compared with an accepted method of thermodilution, the pulmonary artery catheter, in the critically ill patients. Intensive Care Med Exp 2015; 3: 598.

17. Gan H, Cannesson M, Chandler JR, Ansermino JM: Predicting fluid responsiveness in children: a systematic review. Anesth Analg 2013; 117: $1380-1392$.

18. Sobczyk D, Nycz K, Andruszkiewicz P: Bedside ultrasonographic measurement of the inferior vena cava fails to predict fluid responsiveness in the first 6 hours after cardiac surgery: a prospective case series observational study. J Cardiothorac Vasc Anesth 2015; 29: 663-669.

19. Vaish H, Kumar V, Anand R, Chhapola V, Kanwal SK: The correlation between inferior vena cava diameter measured by ultrasonography and central venous pressure. Indian J Pediatr 2017; 84: 757-762.

20. Babaie S, Behzad A, Mohammadpour M, Reisi M: A comparison between the bedside sonographic measurements of the inferior vena cava indices and the central venous pressure while assessing the decreased intravascular volume in children. Adv Biomed Res 2018; 7: 97.

21. Lujan Varas J, Martinez Díaz C, Blancas R, Martnez Gonzalez O, Llorente Ruiz B, Molina Montero R et al.: Inferior vena cava distensibility index predicting fluid responsiveness in ventilated patients. Intensive Care Medicine Experimental 2015; 3 (Suppl 1): A600.

22. Rahman NH, Ahmad R, Kareem MM, Mohammed MI: Ultrasonographic assessment of inferior vena cava/abdominal aorta diameter index: a new approach of assessing hypovolemic shock class 1. Int J Emerg Med 2016; 9: 8 .
23. Kircher BJ, Himmelman RB, Schiller NB: Noninvasive estimation of right atrial pressure from the inspiratory collapse of the inferior vena cava. Am J Cardiol 1990; 66:493-496.

24. Mannarino S, Bulzomì P, Codazzi AC, Rispoli GA, Tinelli C, De Silvestri A et al.: Inferior vena cava, abdominal aorta, and IVC-to-aorta ratio in healthy Caucasian children: Ultrasound Z-scores according to BSA and age. J Cardiol 2019; 74: 388-393.

25. Taneja K, Kumar V, Anand R, Pemde HK: Normative data for IVC diameter and its correlation with the somatic parameters in healthy indian children. Indian J Pediatr 2018; 85: 108-112.

26. Kathuria N, Ng L, Saul T, Lewiss RE: The baseline diameter of the inferior vena cava measured by sonography increases with age in normovolemic children. J Ultrasound Med 2015; 34: 1091-1096.

27. Kutty S, Li L, Hasan R, Peng Q, Rangamani S, Danford DA: Systemic venous diameters, collapsibility indices, and right atrial measurements in normal pediatric subjects. J Am Soc Echocardiogr 2014; 27: 155162.

28. Li DK, Wang XT, Liu DW: Association between elevated central venous pressure and outcomes in critically ill patients. Ann Intensive Care 2017; 7: 83-89.

29. Marik PE, Cavallazzi R: Does the central venous pressure predict fluid responsiveness? An updated meta-analysis and a plea for some common sense. Crit Care Med 2013; 41: 1774-1781.

30. Natori H, Tamaki S, Kira S: Ultrasonographic evaluation of ventilatory effect on inferior vena cava configuration. Am Rev Respir Dis 1979; 120: 421-427.

31. Mugloo MM, Malik S, Akhtar R: Echocardiographic inferior vena cava measurement as an alternative to central venous pressure measurement in neonates. Indian J Pediatr 2017; 84: 751-756.

32. Long E, Duke T, Oakley E, O'Brien A, Sheridan B, Babl FE, Pediatric Research in Emergency Departments International Collaborative (PREDICT): Does respiratory variation of inferior vena cava diameter predict fluid responsiveness in spontaneously ventilating children with sepsis. Emerg Med Australas 2018; 30: 556-563.

33. Orso D, Paoli I, Piani T, Cilenti FL, Cristiani L, Guglielmo N: Accuracy of ultrasonographic measurements of inferior vena cava to determine fluid responsiveness: a systematic review and meta-analysis. J Intensive Care Med 2020; 35: 354-363.

34. Tan HL, Wijeweera O, Onigkeit J: Inferior vena cava guided fluid resuscitation - fact or fiction? Trends Anaesth Crit Care 2015; 5: 70-75.

35. Bilgili B, Haliloglu M, Tuğtepe H, Umuroglu T: The assessment of intravascular volume with inferior vena cava and internal jugular vein distensibility indexes in children undergoing urologic surgery. J Invest Surg 2018; 31: 523-528.

36. Barbier C, Loubières Y, Schmit C, Hayon J, Ricôme JL, Jardin F et al.: Respiratory changes in inferior vena cava diameter are helpful in predicting fluid responsiveness in ventilated septic patients. Intensive Care Med 2004; 30: 1740-1746.

37. Basu S, Sharron M, Herrera N, Mize M, Cohen J: Point-of-care ultrasound assessment of the inferior vena cava in mechanically ventilated critically ill children. J Ultrasound Med 2020.

38. Mende R, Oliveira MV, Padilha GA, Santos RS, Rocha NN, Luiz RR et al.: Distensibility index of the inferior vena cava in experimental acute respiratory distress syndrome. Respir Physiol Neurobiol 2017; 237: 7-12. 